\title{
Recent advances in novel functional nanomaterials-based oil/water separation techniques
}

\author{
Li Sen ${ }^{1}$, Ji Yunzhe ${ }^{1}$, Li Xiaojing ${ }^{1}$ and Wang Xiaojie ${ }^{1, *}$ \\ ${ }^{1}$ Naval Logistics Academy, 300450 Tianjin, China
}

\begin{abstract}
The increasing amount of oily wastewater discharges caused major challenges for current environmental issues, such as oil-water purification and separation ${ }^{[1-6]}$. Thus, the development of new functional materials for the efficient treatment of oil-water is imperative [7-11]. This paper reviewed the recently developed oil-water separation technologies, which were based on novel functional nanomaterials, and presented representative works in detail. Finally, the challenges and future research directions of this area were briefly discussed.
\end{abstract}

\section{Introduction}

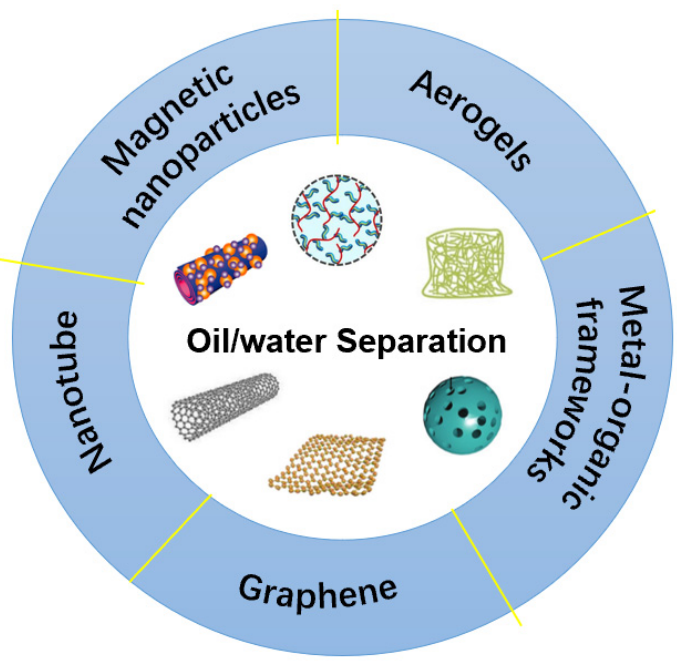

Scheme 1. Schematic illustration of nanomaterials-based oil/water separation techniques.

Oily wastewater contains suspended oil, dispersed oil, emulsified oil, and dissolved oil (nano-level), which are classified based on the size of oil droplets in water ${ }^{[12-13]}$. Owing to the larger particle size, suspended oil and dispersed oil tend to agglomerate into a continuous oil layer, which could be easily removed by traditional methods, such as adsorption, sedimentation, and mechanical oil skimming. In order to obtain more stable emulsified oil and dissolved oil, various traditional oilwater separation techniques (dispersant, air floatation, sedimentation, biotechnology and ultra/microfiltration membrane separation) have been applied to treat oily wastewater [14-17]. However, the energy consumption and treatment efficiency are poor. Among various methods, physical adsorption is one of the most common and effective methods owing to the simple operation and low cost, while the key to the adsorption method is the processing efficiency and applicability of adsorbent materials [18-25]. The actual application of traditional adsorption materials is limited due to high cost, poor adsorption performance, low oil recovery, and adsorption saturation sinking. Besides, the adsorption materials are difficult to recover, which further produces secondary environmental pollution. Therefore, the development of new adsorption and separation materials with low cost, simple preparation method, high separation efficiency, and easy recovery is the key to realize oil-water separation ${ }^{[26]}$.

Nanomaterials exhibit strong adsorption properties due to their larger specific surface area and more surface-active groups ${ }^{[27]}$. In addition, nanostructured materials exhibit a faster mass transfer process for pollutants and achieve rapid adsorption or degradation of pollutants. Thus, nanomaterials show great potential for the preparation of high-performance adsorbents. It is foreseeable that the development of new functional nanomaterials will lead to breakthrough changes in traditional oil-water separation technology.

\section{Oil/water separation technology based on functional nanomaterials}

\subsection{Graphene-based nanomaterials}

Graphene, a three-dimensional network structure material received a lot of attention owing to the larger specific surface area, better hydrophobic properties, and stronger adsorption capacity ${ }^{[28]}$. However, the toughness of these materials is below the level required for practical applications. Therefore, these materials will be

\footnotetext{
* Corresponding author: lisen202009@ foxmail.com
} 
(a)

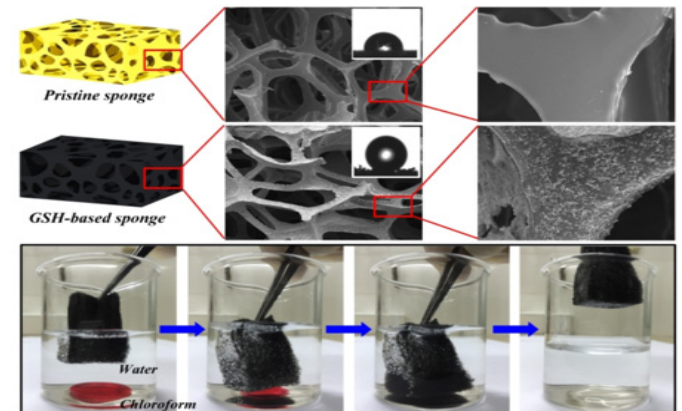

(b)

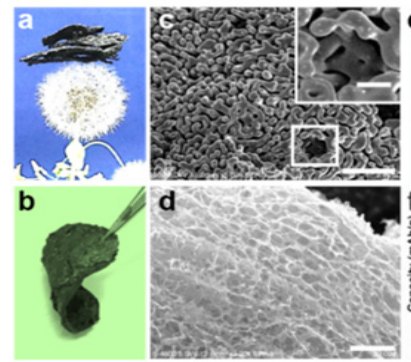

(c)

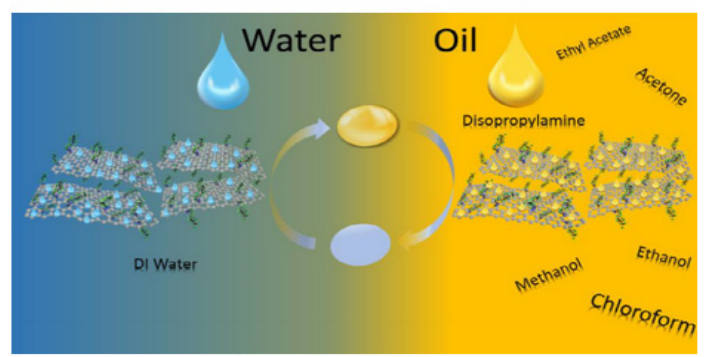

Figure 1. Graphene-based nanomaterials for oil-water separation. (a) Thiolated graphene-based superhydrophobic sponges for oil-water separation ${ }^{[32]}$; (b) shell-based threedimensional (3D) graphene foam ${ }^{[33]}$; (c) L-ascorbic acid (LA) based reduced graphene oxide $(\mathrm{rGO})^{[34]}$.

damaged by the external forces after oil-water separation and lose the recycling function. Many researchers developed oil-water separation materials using graphene. For example, Bi et al. prepared nano-porous graphene sponges through a hydrothermal reduction method ${ }^{[29]}$. Nguyen et al. ${ }^{[30]}$ prepared superhydrophobic sponges for oil-water separation using a dip-coating-drying method by adsorbing reduced graphene oxide onto polyurethane sponges. Zhou et al. [31] prepared superhydrophobic sponges based on trimethoxysilane modified with graphene using the one-pot method. However, these studies were only limited to graphene, reduced graphene oxide, and functionalized graphene modified with silane coupling agents.

Zeng et al. ${ }^{[32]}$ proposed a method to prepare superhydrophobic polyurethane sponges for oil-water separation. The graphene oxide (GO) was first reduced to thiolated graphene (GSH), which exhibited a water contact angle of $128^{\circ}$. Then the sponge was soaked in ethanol and dried to prepare a superhydrophobic sponge. The WCAs of the as-prepared sponges were largely determined by the loading of GSH, and the WCAs reached $157.0^{\circ}$ and $160.5^{\circ}$ with GSH loadings at $5.0 \%$ and $7.5 \%$, respectively. The oil absorption experiments demonstrated that it could quickly absorb various organic solvents and oils with an adsorption mass reaching 30 to 90 times compared with its own weight. Besides, the absorption capacities for crude oil all the organic and oils were above 29.5 times its own weight. The preparation process is simple and the cost is low, which exhibits a great practical application prospect. Liu et al. ${ }^{[33]}$ prepared a three-dimensional (3D) graphene foam via the shell-based CVD technique. The graphene sheets in the resulting foam were seamlessly connected into a three-dimensional flexible network, forming highly porous materials with negligible non-carbon impurities, ultra-low density, excellent mechanical flexibility, and electrical conductivity. These 3D graphene foams exhibited fast adsorption performance on various oils and organic solvents with an adsorption capacity of 250 times compared with the original material weight. The shell-derived 3D graphene foam exhibited excellent mechanical flexibility and electrical conductivity, and the graphene foam also showed rapid adsorption of various oils and organic solvents with an adsorption capacity of 250 times compared with the original material weight. Zeng et al. ${ }^{[34]}$ reported a novel, facile, environmentally friendly, and economical synthesis method of amphiphilic LA/F/rGO hydrogels, which was prepared in the presence of reducing agent Lascorbic acid (LA) based on a low-temperature hydrothermal process of partially reduced graphene oxide (rGO) with good oil-repellent and oil-elastic properties underwater. The density was $19 \mathrm{mg} / \mathrm{ml}$, and it exhibited excellent thermal stability and regenerable adsorption capacity (up to 20 times its original weight) to various organic solvents. The prepared LA/F/rGO hydrogel exhibited a spontaneous repelling effect on oil droplets underwater. The oil droplets were bounced off from the hydrogel material underwater. The novel Lascorbic acid (LA)/F/rGO hydrogels provided a new perspective for the development of new graphene-based nanocomposites for oil-water separation and oil spills, which exhibited wide engineering implications.

\subsection{Nanotubes-based nanomaterials}

Chen et al. ${ }^{[35]}$ prepared a layered composite membrane, which consisted of a submerged superoleophobic carbon nanotube membrane and a core-shell polystyrene $@ \mathrm{Au}$ nanoparticle assembly membrane (Figure.1(a)). It achieved simultaneous flow-through degradation of miscible toxic organic molecules in water and oil-water emulsion. The multifunctional composite membrane can continuously treat contaminated water. This multifunctional composite membrane is a promising candidate for water purification owing to the capacity of continuous treatment of polluted oil-containing wastewater. The multifunctional hierarchical CNTs/PS@AuNPs composite membranes were prepared by catalytic degradation of soluble organic molecules and oil/water emulsion separation. The obtained catalytic The obtained catalytic composite membranes with the submerged superoleophobic surface achieved 92.6\% decomposition of nitrophenols in oil and water, degradation of water-soluble organic pollutants, and oil/water emulsion separation through only one device. 
Wang et al. ${ }^{[36]}$ proposed a method to prepare carbon nanotube-reinforced polyurethane sponges with superhydrophobic and lipophilic properties. The method involved oxidative autopolymerization of dopamine and the reaction of hydrophilic polydopamine (PDA) with hydrophobic octadecylamine. The superhydrophobic stability of the sponges was investigated. The prepared sponges rapidly and selectively adsorbed various oils and fats with an adsorption ratio of 34.9 times compared with their own weight, which could be collected via a simple extrusion process. More interestingly, the mechanical strength of the prepared sponges was improved due to the structural enhancement of CNTs anchored on the sponge skeleton. The recovered sponge

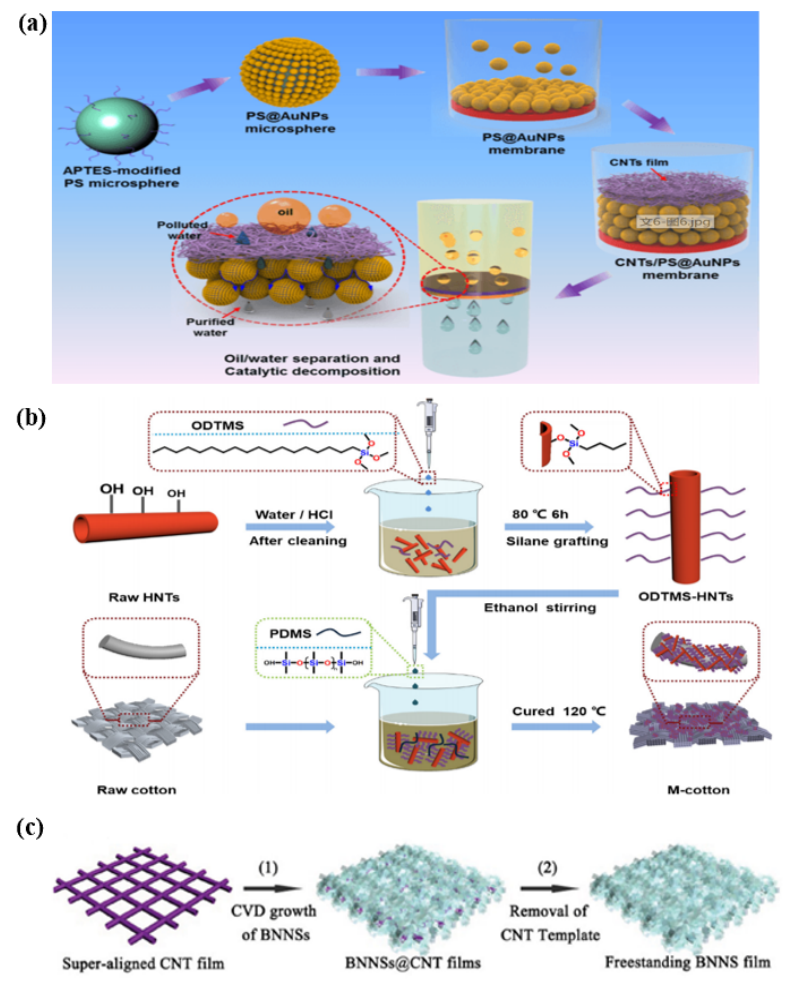

Figure 2. (a) CNTs/PS@AuNPs composite membranes ${ }^{[35]}$; (b) ODTMS-HNTs on a stainless-steel mesh ${ }^{[41]}$; (c) boron nitride nanotube (BNNT) coated networks ${ }^{[44]}$.

was reused 150 times for the separation of oil-water mixtures. After that, it still maintained a high absorption rate. This multifunctional sponge showed great potential for large-scale oil-water separation. Wang et al. ${ }^{[37]}$ added $100 \mathrm{mg}$ carbon nanotubes (CNTs) to dopamine solution with $\mathrm{pH}=8.5$, which was further ultrasonically dispersed to load polydopamine films on the surface of CNTs. Finally, it was dried to produce CNT-PDA powder loaded with polydopamine coating. The polyurethane sponge $(15 \mathrm{~mm} \times 10 \mathrm{~mm} \times 10 \mathrm{~mm})$ after ultrasonic dispersion was cleaned with ethanol aqueous solution and then immersed in a strong acid solution of $\mathrm{CrO}_{3}$ for $1 \mathrm{~min}$, which was further added to alkaline dopamine solution together with CNT-PDA and stirred for $24 \mathrm{~h}$. CNT-PDA was targeted and fixed on the polyurethane sponge skeleton through the self-polymerization reaction of dopamine moieties ${ }^{[38-40]}$.
HNTs are typical layered silica-aluminate minerals with hollow tubular morphology, which are abundant, low-cost, and have good biocompatibility. Thus, they were widely used in catalytic, clinical, electronic, and separation fields. Guo et al. [41] prepared a superhydrophobic stainless steel mesh by spraying epoxy resin and octadecyltrimethoxysilane-modified HNTs on a stainless-steel mesh, which exhibited a separation efficiency of more than $98 \%$ for oil-water mixtures. Zeng et al. fabricated a novel oil-water separation membrane by APTES-modified HNTs, which exhibited excellent oil barrier properties. Zhang et al. ${ }^{[42]}$ reported a superhydrophobic fabric based on HNTs was prepared by a simple impregnation-drying process, which had a strong resistance to water and a high affinity for oil (Figure 1(b)). The superhydrophobic cotton fabric was first prepared by loading HNTs onto the surface of the fabric by a cyclic impregnation-drying method using octadecyltrimethoxysilane-modified elosite nanotubes (ODTMS-HNTs) as the material. The superhydrophobic fabric removed the oil by the adsorption method, which could adsorb and separate the floating oil with a density less than water and the sunken oil with a density greater than water. The adsorption amount and separation efficiency reached 12.5 times compared with its own mass and 99.99\%, respectively. In addition, the superhydrophobic fabric still exhibited excellent separation after 10 cycles. High-performance oil-water separation devices made of environmentally friendly materials are a promising strategy to solve water pollution problems. Liu Group developed an oil-water separation system using chitin chitin/erolite nanotubes (C/HNTs) as the composite materials ${ }^{[43]}$. C/HNTs were cross-linked with epichlorohydrin and lyophilized, forming porous sponges. The C/HNTs sponges were modified into hydrophobic sponges by immersing them in bromohexadecane ethanol solution. The surface structure, mechanical properties, microstructure, oil absorption properties, and oil-water separation properties of the $\mathrm{C} / \mathrm{HNTs}$ sponges were investigated. Infrared spectra showed that bromomethyl was successfully coated on the surface of the C/HNTs sponge, leading to a hydrophilic and lipophilic structure. The internal structure of the C/HNTs sponge was filled with interconnected pores with a pore size of $250 \sim 500 \mu \mathrm{m}$. The adsorption properties of oils and fats, such as toluene, sunflower oil, chlorocarbon, n-hexane, chloroform, and acetone were measured. The results showed that the total absorption of chloroform by the C/HNTs sponge was 11.23 times compared with the weight of the sponge, and the total absorption of hexane was 3.94 times compared with the weight of the sponge. The sponge also showed good oil-water separation ability with a separation efficiency of $98.7 \%$. The results indicated that the $\mathrm{C} / \mathrm{HNT}$ s sponge with good mechanical properties had great potential for oil-water separation applications.

Two-dimensional boron nitride nanosheets (BNNSs) received much attention because of their excellent properties and potential applications. Because the $\mathrm{sp}^{2}$ bonded structure was similar to that of graphene, the 
mechanical properties and thermal conductivity of BNNSs were comparable to those of graphene. In addition, BNNSs exhibited high oxidation resistance, superhydrophobicity, and UV-emitting properties. All these properties made them very attractive composite materials, thermally conductive materials, protective coatings, and oil-water separation materials. Yu et al. ${ }^{[44]}$ developed a simple, effective, and scalable method to fabricate boron nitride nanotube (BNNT) coated networks with superhydrophobicity and superoleophilicity. The BNNT with diameters of $100 \sim$ $400 \mathrm{~nm}$ was partially arranged vertically on the surface of the stainless-steel mesh, which changed the surface properties of the stainless-steel mesh from hydrophobic with high CA hysteresis to superhydrophobic and superoleophilic with no hysteresis. The experimental results showed that the oil-water separation efficiency remained basically unchanged and exhibited no obvious deterioration after more than 100 cycles. This indicated that BNNT coated network not only exhibited superhydrophobicity and super lipophilicity but also exhibited the advantages of high stability, durability, and repeatability. Considering the good mechanical and chemical stability of BNNT, the BNNT-coated stainlesssteel meshes were a promising candidate for oilcontaminated water treatment. Yao et al. ${ }^{[45]}$ prepared a novel BNNS material-independent BNNS film (Figure 1(c)). Super-aligned carbon nanotube (CNT) films were used as templates, and BNNSs were grown at a high density around the CNT bundles. Then, the templates were removed to obtain the freestanding BNNS films. The freestanding BNNS films exhibited excellent hydrophobicity and lipophilicity. These excellent properties made them suitable for oil-water separation with extremely high flux and excellent separation efficiency. The membrane could be used more than 20 times with good stability and reproducibility.

\subsection{Gels}

Aerogels are new materials with a wide range of applications in energy storage, oil absorption, and catalysts due to their abundant properties, low cost, and environmental friendliness. Wang ${ }^{[46]}$ proposed a method for the preparation of nitrogen-rich carbon aerogel (NRC aerogel) with a highly porous structure and nitrogen-rich surface for the efficient separation of specific substances, such as oil and organic contaminants. This NRC aerogel could also be used to collect micron-sized oil droplets from oil-water mixtures with an excellent efficiency that far exceeds that of most existing separation methods. In addition, the NRC aerogel can be used as a recyclable container for oil and chemical cleanup due to its excellent hydrophobic and fire-resistant properties. The carbon aerogels exhibit $61.2 \%$ absorption capacity after 100 absorption/combustion cycles, thus providing the highest recyclability reported in the literature. All these characteristics make these prepared NRC aerogels suitable for a wide range of applications in water purification and treatment. (a)

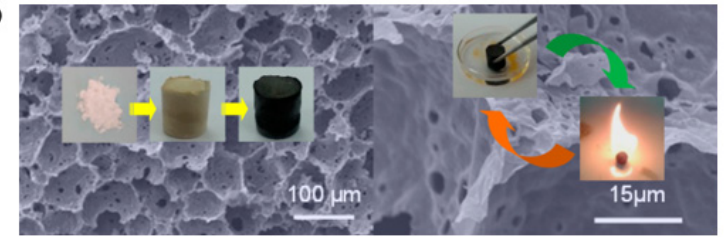

(b)

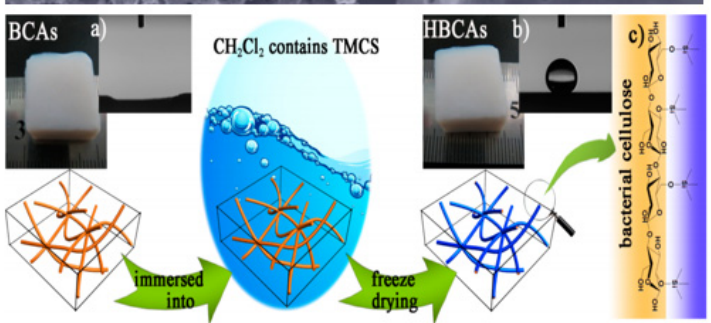

(c)

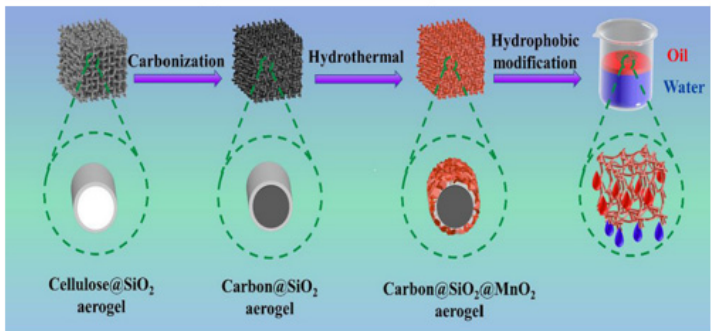

(d)

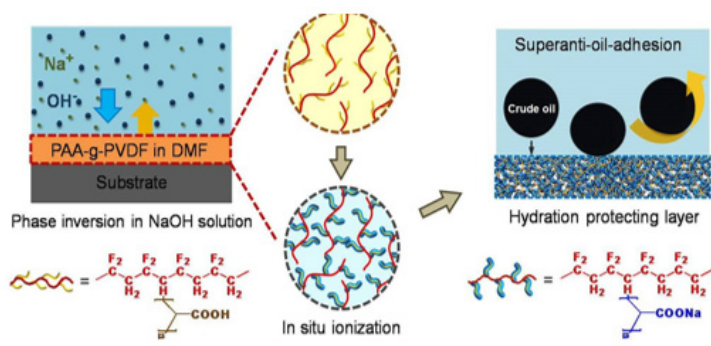

Figure 3. Fabrication and water treatment application of aerogels-based nanomaterials ${ }^{[46-51]}$.

Sai et al. ${ }^{[47]}$ immersed block bacterial cellulose aerogels (BCAs) in an organic solvent containing trimethylchlorosilane (TMCS), trimethylsilylated the three-dimensional meshwork skeleton of BCAs, and then lyophilized them to obtain intact hydrophobic bacterial cellulose aerogels (HBCAs). In contrast to the CVD method, the modifier was uniformly dispersed in the liquid and diffused rapidly into the pores with the dispersant. This process ensured the same reaction environment of all the cellulose nanofibers in the aerogel, thus avoiding the inhomogeneity of the material obtained by the CVD method. HBCA exhibited hydrophobic and lipophilic properties, which made it suitable for the selective adsorption of oil in water. Besides, HBCA exhibited a wide range of absorption of grease and organic solvents with the highest adsorption capacity of 185 times compared with its own weight.

Qiu et al. [48] prepared a compressible, superhydrophobic, multifunctional graded biomass carbon@SiO $\mathrm{Si}_{2} @ \mathrm{MnO}_{2}$ aerogel (HBCSM aerogel) was successfully using economical and environmentally friendly sisal cellulose as the main raw material. The cellulose@ $\mathrm{SiO}_{2}$ aerogel (CS aerogel) was prepared by the sol-gel method, followed by charring to obtain biomass carbon@ $\mathrm{SiO}_{2}$ aerogel (BCS aerogel). Then, HBCSM aerogels were prepared by in situ assembly of 
$\mathrm{MnO}_{2}$ nanosheets on the surface of BCS aerogels under hydrothermal conditions. The incorporation of graded $\mathrm{SiO}_{2} @ \mathrm{MnO}_{2}$ structure into the HBCSM aerogel not only improved the surface roughness and hydrophobic properties of the aerogel, but also improved the absorption capacity and mechanical durability.

Jin et al. ${ }^{[50]}$ organically combined hydrogel materials with strong water absorption and hydrophilic properties with traditional polymeric film-forming materials and successfully prepared high-strength ionized hydrogel polymeric membrane materials with superhydrophobic, zero-adhesion, anti-pollution and self-cleaning properties for crude oil, heavy oil and other high-viscosity oils in water. The material was prepared from poly (acrylic acid)-grafted poly(vinylidene fluoride) (PAA-g-PVDF) by the alkali-induced phase transformation method. The PVDF-grafted PAA underwent in situ ionization to form a hydrogel region with a stronger hydration capacity of sodium polyacrylate (PAAS), thus imparting the PAASg-PVDF membrane with excellent superhydrophobic and zero oil adhesion properties in water. PAAS-g-PVDF exhibited an oleophobic angle of more than $165^{\circ}$ in water and almost zero oil adhesion to high viscosity oils such as crude oil and heavy oil. PVDF was in contact with crude oil in the air. When it is put into water, the crude oil was automatically detached and achieved selfcleaning. This coating material can be used for oil-proof clothing and oil-proof facilities required in cases of crude oil spills, and has great application prospects.

Separation of oil-water mixtures in highly acidic, highly alkaline, and highly saline environments is a great challenge. There is an urgent need to prepare novel materials for efficient oil-water separation in highly acidic, alkaline, and saline environments. Jiang [51] reported a simple method to prepare stable hydrogelcoated filter paper, which not only could separate oilwater mixtures in highly acidic, alkaline, and salty environment, but also surfactant-stabilized emulsion. The hydrogel-coated filter paper was prepared by crosslinking the filter paper with hydrophilic polyvinyl alcohol through an aldol condensation reaction with glutaraldehyde. The results showed that the hydrogelcoated filter paper could separate oil-water mixtures in highly acidic, alkaline, and saline environments and oilwater emulsion environments with a separation efficiency of more than $99 \%$.

\subsection{Magnetic nanoparticles}

Magnetic nanomaterials could be easily and rapidly separated from aqueous solutions under the action of an applied magnetic field, which is of simplicity, high efficiency, high sensitivity, and low cost. Magnetic nano- $\mathrm{Fe}_{3} \mathrm{O}_{4}$ is an important spinel-type ferrite with a huge specific surface area, excellent magnetic properties, and high surface activity. The attachment of magnetic nanoparticles to other hydrophilic-oleophilic materials after modification of different functional groups could control and recover oil-water separation materials under the action of magnetic fields.
Zhang et al. ${ }^{[52]}$ fabricated $\mathrm{Fe}_{3} \mathrm{O}_{4}$ nanoparticles on the surface of polydopamine particles by combining the oxidation and self-polymerization of dopamine. It was further modified with low-surface-energy materials to create particles with magnetic properties. Finally, the above particles were combined with PU sponges to obtain magnetic sponges with superhydrophobicity and superoleophilicity, which exhibited high oil absorption capacity and increased the adsorption ratio from $1348 \%$ to $7268 \%$. Zhang XL et al. ${ }^{[33]}$ synthesized $\mathrm{Fe}_{3} \mathrm{O}_{4} @ \mathrm{SiO}_{2}$ nanocomposite particles via adding ethyl orthosilicate to $\mathrm{Fe}_{3} \mathrm{O}_{4}$ MNPS, which exhibited a good adsorption effect on organic pollutants, such as perfluorooctanoic acid (PFOA), perfluorononanoic acid (PFNA), etc. Cao HM et al. ${ }^{[54]}$ synthesized $\mathrm{Fe}_{3} \mathrm{O}_{4} @ \mathrm{SA}$ nanoparticles using Lcysteine as a template, which exhibited a remarkable ability to remove oil slick at the water surface and could be easily recycled for multiple uses.

\subsection{MOFs-based nanomaterials}

Metal-organic frameworks (MOFs) have promising applications in many fields such as gas storage and separation, catalysis, and drug delivery. Metal/carbon aerogel materials derived from MOFs were prepared by pyrolysis, which not only maintained the advantage of the large specific surface area of MOFs structure but also exhibited more stable structure and mechanical strength. In addition, most MOFs materials are composed of transition metals ( $\mathrm{Fe}, \mathrm{Co}, \mathrm{Ni}$, etc.), which are usually essential elements in catalytic systems. If the adsorbed organic compounds can be further catalytically transformed to another high value-added product, the secondary utilization of oil-containing pollutants will be realized. Therefore, metal/carbon aerogels assembled from MOFs are expected to be useful for adsorption and catalytic conversion of various oil and organic compounds.

High-performance membranes for simultaneous removal of insoluble emulsified oil and soluble organic dyes are urgently needed for wastewater treatment. Xue ${ }^{[55]}$ prepared a bifunctional membrane with the good antifouling ability for dye/oil/water emulsion separation by growing a flower-like metal-organic backbone (MIL53-OH) on a polyacrylonitrile/polyethyleneimine membrane for the first time. The stable and super strong oil-repellent hydration layer of the membrane was attributed to the synergistic effect of the layered flowerlike structure and the highly hydrophobic and super hydrophilic component, leading to strong oil resistance and excellent oil-water emulsion separation performance (permeate flux $>4000 \mathrm{~L} \mathrm{~m}-2 \mathrm{~h}-1$ ) of the membrane. More importantly, the super hydrophilic component endowed the membrane with good electrostatic and hydrogen-bonding ability for dye removal. The membrane retention rates for both dyes and emulsified oils were above $99 \%$. Su et al. ${ }^{[56]}$ synthesized hydrogels through the instability of Ni-MOFs in water. Subsequently, the Ni/C aerogels mosaic with $\mathrm{Ni}$ nanoparticles was prepared by pyrolytic carbonization under a nitrogen atmosphere. Compared with the 
conventional aerogel preparation process, this preparation process is environmentally friendly and simple. Besides, the material exhibits excellent properties, such as ultra-light, superhydrophobic, highly elastic, and magnetic. These properties give the $\mathrm{Ni} / \mathrm{C}$ aerogel excellent adsorption and catalytic ability. Therefore, the Ni/C aerogel shows excellent adsorption efficiency for various oils and organic compounds (3899\% and $4877 \%$ for motor oil and carbon tetrachloride adsorption, respectively) and good cyclic adsorption stability.

\section{Summary and perspectives}

With the development of nanotechnology, oil-water separation technology based on new nanomaterials has great research potential and application prospects. The basic structure of nanomaterials determines their super adsorption capacity. The fabrication of superhydrophobic-superoleophilic and superhydrophilicsuperoleophobic materials could fully utilize the properties of nanomaterials. The development of new adsorbent materials with low cost, biodegradable or good recycling performance, optimization of the superhydrophobicity and lipophilicity, adsorption capacity and stability under harsh environmental conditions, and more efficient and environmentally friendly oil-water separation materials are the focus of future research.

\section{Acknowledgment}

This work was supported by the National Key Research and Development Program of China (Grant No. 2017YFC0506306).

\section{References}

1. T. Zhang, B. Gu, F. Qiu, X. Peng, X. Yue, D. Yang, Fibers Polym 19, 2195-2202 (2018).

2. Z. Tao, X. Yue, D. Yang, Q. Guo, Z. Li, Applied Organometallic Chemistry 32, e4244 (2018).

3. AK. Fa Rd, T. Rhadfi, G. Mckay, M. Al-Marri, MA, A. K. Hussien, Chemical Engineering Journal 293, 90-101 (2016).

4. M. A. Abdullah, A.U. Rahmah, Z. Man, J. Hazard. Mater 177, 683-691 (2010).

5. G. Kwon, A.K. Kota, Y. Li, A. Sohani, J.M. Mabry, A. Tuteja, Advanced Materials 24, 3666-3671 (2012).

6. Z. Khatib, P. Verbeek, Society of Petroleum Engineers 55, 26-28(2002).

7. Y. Cao, N. Liu, W. Zhang, L. Feng, Y. Wei, ACS Applied Materials \& Interfaces 8, 3333-3339 (2016).

8. C. Lee, B. Tiwari, D. Zhang, Environmental Science Nano 4, 514-525(2017).
9. I. B. Ivshina, M. S. Kuyukina, A. V. Krivoruchko, A. A. Elkin, S. O. Makarov, C. J. Cunningham, T. A. Peshkur, R. M. Atlas, J. C. Philp, Environ. Sci.: Processes Impacts 17, 1201-1219(2015).

10. S. B. Joye, Science 349, 592-593(2015).

11. R. C. Prince, Environmental Science \& Technology 49, 6376-84 (2015).

12. C. Yu, W. Lin, J. Jiang, Z. Jing, P. Hong, Y. Li, RSC Advances 9, 37759-37767(2019).

13. S. Barroso-Solares, J. Pinto, D. Fragouli, A. Athanassiou, Materials 11, 2382(2018).

14. O. Carmody, R. Frost, Y. Xi, S. Kokot, Journal of Thermal Analysis and Calorimetry 91, 809816(2008).

15. I.B. Ivshina, M.S. Kuyukina, A.V. Krivoruchko, A.A. Elkin, J.C. Philp, Environmental Science: Processes and Impacts 17, 1201-1219 (2015).

16.P.Yu, R. Y.Bao, X. J.Shi, W.Yang, M. Yang, Carbohydrate Polymers 155, 507-515 (2017).

17.F. Mapelli, A.Scoma, G.Michoud, F. Aulenta, N.Boon, S.Borin, N.Kalogerakis, D. Daffonchio, Trends in biotechnology 35, 860-870 (2017).

18. L. Yu, H. Yang, Y. Wang, W. Jiang, Powder Technology 311, 257-264 (2017).

19.Z. Tao, L. Kong, Y. Dai, X. Yue, J. Pan, Chemical Engineering Journal 309, 7-14(2017).

20. L.Yan, Q. Li, X. Wang, H. Song, H. Chi, Y. Qiao, Y. Zhai, D. Liu, Polymer-Plastics Technology and Engineering 56, 1857-1865 (2017).

21. J. Rong, F. Qiu, T. Zhang, X. Zhang, Y. Zhu, J. Xu, D. Yang, Y. Dai, Chemical Engineering Journal 322, 397-407 (2017).

22. O. Carmody, R. Frost, Y. Xi, S. Kokot, Journal of Thermal Analysis and Calorimetry 91, 809-816 (2008).

23. S. Zhou, G. Hao, X. Zhou, W. Jiang, T. Wang, N. Zhang, L. Yu, Chemical Engineering Journal 302, 155-162(2016).

24. N. M. Temnikov, Y. N. Kononevich, N. Yuriy, I. B. Meshkov, B. Mikhail I, V.G. Vasil'ev, G. G. Nikiforova, A. M. Muzafarov, Polymer: The International Journal for the Science and Technology of Polymers 138, 255-266 (2018).

25. T. Zhang, D. Yuan, Q. Guo, F. Qiu, D. Yang, Z. Ou, Food and Bioproducts Processing 114, 154-162 (2019).

26. K. C. Taylor, R. A. Burke, H. A. Nasr-El-Din, Journal of Petroleum Science and Engineering 21, 129-139(1998).

27. Y. Zuo, L. Zheng, C. Zhao, H. Liu, Small 16, 1903849 (2020).

28. B. H. Nguyen, V. H. Nguyen, Advances in Natural Sciences: Nanoscience and Nanotechnology 7, 2(2016). 
29. H. Bi, X. Xie, K. Yin, Y. Zhou, S. Wan, L. He, F. Xu, F.Banhart, L. Sun, R. S. Ruoff, Advanced Functional Materials 22, 4401-4401(2012).

30. D. Nguyen, N. Tai, S. Lee, W. Kuo, Energy \& Environmental Science 7, 7908-7912(2012).

31.S. Zhou, G. Hao, X. Zhou, W. Jiang, T. Wang, N. Zhang, L. Yu, Chemical Engineering Journal 302, 155-162 (2016).

32. L. Zhang, H. Li, X. Lai, X. Su, T. Liang, X. Zeng, Chemical Engineering Journal 316, 736-743 (2017).

33. L. Shi, K. Chen, R. Du, A. Bachmatiuk, M. H. Ruemmeli, K. Xie, Y. Huang, Y. Zhang, Z. Liu, Journal of the American Chemical Society 138, 6360 (2016).

34. W. Hu, P. Zhang, , X. Liu, B. Yan, L.Xiang, , J. Zhang, L. Gong, L. Jiang, Chemical Engineering Journal 353, 708-716 (2018).

35. L. Zhang, J. Gu, L. Song, L. Chen, Y. Huang, T.Chen, Journal of Materials Chemistry A 4, 1081010815 (2016).

36. H. Wang, E. Wang, Z. Liu, D. Gao, R. Yuan, L. Sun, Y. Zhu, Journal of Materials Chemistry A 3, 266273 (2014).

37. S. Wang, X. Peng, L. Zhong, J. Tan, S. Jing, X. Cao, W. Chen, C. Liu, R. Sun, Journal of Materials Chemistry A 3, 8772-8781(2015).

38. J. Huang, E. Cho, Y.S. Hsiao, K. C. Lee, RSC Advances 5, 53741-53748(2015).

39. J. Song, Y. Lu, J. Luo, S. Huang, L. Wang, W. Xu, I. P. Parkin, Advanced Materials Interfaces 2, 350358(2015).

40. T. Zhang, B. Gu, F. Qiu, X. Peng, X. Yue, D. Yang, Fibers and Polymers 19, 2195-2202(2018).

41. D. Guo, J. Chen, K. Hou, S. Xu, J. Cheng, X. Wen, S. Wang, C. Huang, P. Pi, APPLIED CLAY SCIENCE 156, 195-201(2018).

42. Q. Song, H. Wang, S. Han, J. Wang, B. Zhang, Y. Zhang, Progress in Organic Coatings 148, 105839 (2020).

43.X. Zhao, Y. Luo, P. Tan, M. Liu, C. Zhou, International Journal of Biological Macromolecules 132, 406-415 (2019).

44. Y. Yu, H. Chen, Y. Liu, V. Craig, L. Li, Y. Chen, Advanced Materials Interfaces 1, 1300002 (2014).

45. T. Li, L. Wang, Z. Kai, Y. Xu, Y. Yao, Small 12, 4960-4965 (2016).

46. Y. Yu, T. Zhen, T. Ngai, C Wang, Acs Appl Mater Interfaces 6, 6351-6360 (2014).

47. H. Sai, R. Fu, L. Xing, J. Xiang, Z. Li, F. Li, T. Zhang, Acs Applied Materials \& Interfaces 7, 7373 (2015).

48. T. Zhang, Q. Guo, F. Qiu, D. Yang, Chemical Engineering Journal 351,622-630 (2018).

49. Y. Si, Q. Fu, X. Wang, J. Zhu, J. Yu, G. Sun, B. Ding, S. Gang, ACS Nano 9, 3791-9(2015).
50. S. Gao, J. Sun, P. Liu, F. Zhang, W. Zhang, S. Yuan, J Li, J. Jin, Advanced Materials 28, 5307-5314 (2016).

51. J. Fan, Y. Song, S. Wang, J. Meng, G. Yang, X. Guo, L. Feng, L. Jiang, Advanced Functional Materials 25, 5368-5375 (2015).

52.Z. Liang, L. Li, Z. Dang, Journal of Colloid \& Interface Science 463, 266-271 (2016).

53. X. Zhang, H. Niu, Y. Pan, Y. Shi, Y. Cai, Journal of Colloid and Interface Science 362, 107-112 (2016).

54. H. Cao, J. Li, Y. Shen, Applied Surface Science 301, 244-249 (2014).

55. H Li, L Zhu, X Zhu, M Chao, Q Xue, Journal of Hazardous Materials 408, 124444 (2020).

56. Y. Su, Z. Li, H. Zhou, S. Kang, G. Wang, Chemical Engineering Journal 402, 126205(2020). 